\title{
Energy Efficiency Evaluation of Wake-Up Radio Based MAC Protocol for Wireless Body Area Networks
}

\author{
Zafar Hussain \\ Centre for Wireless Communications \\ University of Oulu \\ Oulu, Finland \\ zafar.malik87@gmail.com
}

\author{
Heikki Karvonen \\ Centre for Wireless Communications \\ University of Oulu \\ Oulu, Finland \\ heikki.karvonen@oulu.fi
}

\author{
Jari Iinatti \\ Centre for Wireless Communications \\ University of Oulu \\ Oulu, Finland \\ jari.iinatti@oulu.fi
}

\begin{abstract}
In this paper, a generic wake-up radio (WUR) based medium access control (MAC) protocol (GWRMAC) has been evaluated from the energy consumption point of view. GWR-MAC protocol's goal is to achieve energy efficiency by avoiding idle listening since the sensor nodes are awakened only when there is a need for communication. A successful wake-up signal (WUS) transmission and reception is needed to awaken the WUR enabled node(s) from the sleep mode which must be taken into account in the energy consumption evaluation. Success of wake-up process depends on the physical layer detection of the signal, and also on the collision probability of the wake-up signals if there can be multiple nodes that are transmitting the WUS approximately at the same time. The success probability of GWR-MAC wake-up process is analyzed in this work taking into account physical and MAC layer aspects when assuming that wake-up signals are transmitted using Aloha channel access method. WUS success probability derivation is incorporated to energy consumption model and results are obtained to compare the total energy consumption of WUR based network and duty -cycling based network. The results show that the WUR based networks can improve energy efficiency in comparison to conventional duty cycling approach when taking into account also the error probability of the wakeup process. Results show also that in which conditions the duty-cycling approach should be preferred instead of WUR approach
\end{abstract}

Keywords-wake-up receiver, medium access control, WBAN, wireless sensor network, energy consumption.

\section{INTRODUCTION}

Wireless body area networks (WBAN) have recently attracted a great deal of attention due to their unique capabilities and promising applications in areas like healthcare, sports, fitness, military and security [1],[2]. In the healthcare domain, WBAN promises to revolutionize healthcare system through allowing inexpensive, unobtrusive, non-invasive, ambulatory monitoring of human's health-status anytime, anywhere. WBANs are supposed to operate properly for long duration of time without any battery recharge or replacement, especially in the in-body (implanted) sensors case. Therefore, energy consumption management is one of the major concerns for WBAN protocols to enable that recharging and replacement of batteries is as infrequent as possible and network can be responsive for prolonged period.

Medium access control (MAC) layer is important from the communications energy consumption point of view. Several MAC protocols have been developed with the energy efficiency design goal. Most of the energy is wasted during communication process because of the collision of packets, idle listening, over hearing, over-emitting, control packet overhead and erroneous detection due to channel fluctuation [3]. In this work the focus is on idle listening which is typically reduced through duty cycling. That is widely used in wireless sensor networks (WSN) and WBANs to save energy by scheduling nodes' sleep and awake periods. However, duty cycling leads to an energy-latency trade-off issue. With a higher duty cycle the packet delay can be shortened but then the energy consumption increases due to longer active period. On the other hand, with a lower duty cycle, the node will consume less energy due to longer sleep period at the cost of higher latency for data delivery.

To mitigate against this energy and latency trade-off, a wake-up radio (WUR) [4], [5] provides a solution, as this is an on-demand approach where the node is woken up by the wakeup signal (WUS). A WUS triggers a node to wake up from the sleep mode to start reception/transmission activities. The WUS is received or sent by a secondary radio transceiver, which consumes extremely low power. The energy benefit of using wake-up radio in comparison with duty-cycling is that nodes do not waste energy on idle listening of the main radio, since they are only awakened when there is a request for communication. The latency benefit is that since wake-up radio is purely ondemand approach, the only delay is the one introduced by the wake-up process itself.

The aim of this research work is to evaluate a generic wakeup radio based MAC (GWR-MAC) protocol energy efficiency [6] taking into account both physical layer (PHY) and medium access control (MAC) layer. The PHY layer analysis includes WUS transmission and its effects in terms of false alarm and miss detection. The MAC layer analysis contains channel access effect to wake-up process success probability. These 
probabilities are then integrated to energy consumption model proposed in [7], to compare WUR based networking and conventional duty cycle management (DCM) based networking. The energy consumption of the networks is calculated by varying several aspects such as wake-up signal-to-noise ratio (SNR), WUS decision threshold and energy per bit-to-noise ratio.

\section{RELATED WORK}

In this section will be introduced relevant WUR solutions and MAC protocols which are based on the WUR usage to save energy in low-power networks such as WSNs and WBANs. A number of approaches have been introduced to design lowpower WUR technologies which can be used to increase the lifetime of the sensor networks. In [8], the authors have introduced a radio-triggered power management where a radiotriggered circuit, is connected to one of the interrupt inputs of the processor and it is independent of any other components on the node except that it needs to activate the wake-up interrupt. This wake-up mechanism is quite simple and energy efficient. The main drawback, despite its very low power consumption, is that it is not robust against false wake-up since this solution cannot accurately differentiate between a noisy channel and a real wake-up signal. In [9] authors have presented an efficient technique for performing energy detection while maintaining high level of performance with low-precision and low-power elements. This technique improves sensitivity of an ultra-low power wake-up receiver which reduces wake-up latency and saves energy. Another approach was proposed in [10], where the researchers presented the design of a WUR receiver which uses a comparator and an ultra-low power microcontroller as active components. They have implemented a nanowatt WUR to generate interrupts and receive data to achieve low power. Another important development was the idea of a Nano WUR [11], where a charged pump, i.e., two stage voltage doublemultiplier, detects the received signal envelope. Then by using a comparator, a correct bit sequence of a received packet is formed. The authors used active filtering for the signal, which decreases energy efficiency of this solution. Several MAC protocols based on WUR usage have been proposed to overcome the energy efficiency challenge [4], [5]. One of them is solution proposed in [12] where authors presented a topology design where all sensors are within range of each other. They used two radios, the main radio and an additional radio which has very low power consumption. Two channels are used: primary and wake-up. The primary channel is used for control packets and sending data while the wake-up channel is used to wake up neighbors. But this approach results in idle listening as the main radio stays on predetermined duration even though there is no ongoing communication.

The protocol we are going to evaluate in this research work is GWR-MAC [6] which is a general level framework suitable for short range communication by taking advantage of the WUR. It has a bidirectional wake-up procedure between sink and sensor nodes. It also allows different channel access methods usage in the transmission period and can be used for different WSN and WBAN applications, while enabling energy efficiency and low-latency communications by utilizing the proposed wake-up procedure, which activates the targeted nodes only when needed. This research work is conducted to evaluate performance of GWR-MAC taking into account the wake-up process success probability at the physical layer. Probabilities of WUS miss detection and false alarm, and successful wake-up transmission during channel access using Aloha method have been derived and they are exploited to calculate energy consumption of the network. By using this approach, a good insight, about in which conditions WUR based approach should be preferred, can be acquired.

\section{SYSTEM MODEL}

The general architecture of a WBAN, as part of the remote healthcare monitoring system, is given in Fig. 1 [13]. In this work, the focus is on the WBAN, where nodes are equipped with two radios, and it comprises a two-tier topology. The lower-tier sensor nodes which are continuously sensing and the higher-tier hub node which is collecting the data from sensor nodes and acts as a gateway to external network(s). The sensor nodes can continuously monitor human vital functions such as blood pressure, heart rate, temperature, sugar level, electrocardiogram (ECG) and respiration rate. The higher tier node will collect data from lower tier node and can trigger different type of actions, depending on the application.

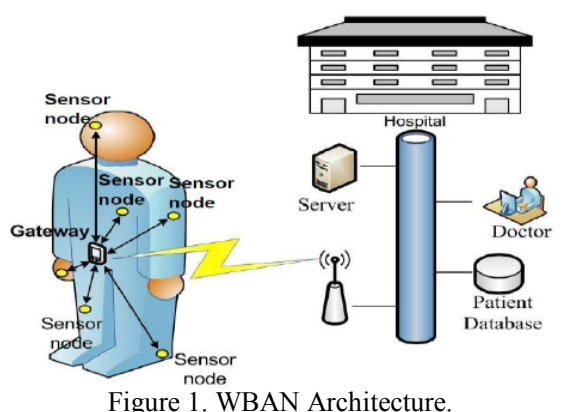

Wake-up procedure for GWR-MAC [6] comprises of two different modes. In source initiated mode the lower-tier sensor nodes transmit a WUS to wake up higher-tier sink node from sleep mode. Upon reception of a WUS, WUR of the sink node will trigger a wake-up through the microcontroller unit (MCU) to its main radio. The main radio of the sink node will broadcast a beacon message (BC) to initiate the transmission period for sensor node as per the channel access procedure. When the sink receives WUS it transmits a $\mathrm{BC}$ signal as an acknowledgement (ACK) to the sensor node. Upon the reception of the $\mathrm{BC}$, the sensor node transmits data to the sink node using channel access method specified in the BC [6]. In sink initiated mode, the sink node will generate a WUS using either broadcast, unicast or multicast signal to wake up sensor nodes. On the reception of a WUS, the sensor node transmits an ACK message to the sink. When the sink node receives an ACK it sends the BC containing information about the following transmission period. These processes have been described in detail in [6]. 
In this work, the focus is on the source-initiated mode. However, the analytical model is applicable also for the sinkinitiated mode with slight modifications. The WUR of the WBAN nodes is assumed to be deployed using the energy detection (ED) based solution introduced in [9]. The data packets are assumed to be communicated using impulse radio ultra wideband (IR-UWB) technique as in [7].

\section{ANALYTICAL MODEL}

Analytical model is developed to take into account the wake-up process success probability in the energy efficiency evaluation of GWR-MAC based network. The analytical model used for wake-up process success derivation is based on the model introduced in [9] and it will be shortly introduced next.

The probability of false alarm, $P_{f}$, is the probability for an output sample at receiver to be larger than the threshold value in the presence of noise only. The probability of miss detection, $P_{m}$, is the probability that the output sample, is less than the threshold value when the signal to be detected, is present. The input signal at the ED detector can be modeled as

$$
x(t)=h *_{s}(t)+n(t)
$$

where $s(t)$ is the WUS signal waveform, $n(t)$ is the noise waveform and $h=1$ or 0 depending is the WUS signal present or not, respectively. Integrator output y will be used to find out is the signal present at the receiver. If the signal is not present $(h=0)$, the integrator output is

$$
y_{0}=\int_{0}^{T} x_{0}^{2}(t) d t=\int_{0}^{T} n^{2}(t) d t
$$

If the signal is present, $\mathrm{y}$ is as follows

$$
y_{1}=\int_{0}^{T} x_{l}^{2}(t) d t=\int_{0}^{T}(s(t)+n(t))^{2} d t
$$

A sampling function of duration $T$, with a bandwidth $B$ can be described by a set of sample values in $2 B T$. Therefore, $y_{0}$ and $y_{1}$ can be approximated as [9]

$$
\begin{aligned}
& y_{0}=\frac{N_{0}}{2} \sum_{i=i}^{2 B T}\left(\frac{a_{i}}{\sqrt{N_{0} B}}\right)^{2}, \\
& y_{1}=\frac{N_{0}}{2} \sum_{i=i}^{2 B T}\left(\frac{a_{i}+b_{i}}{\sqrt{N_{0} B}}\right)^{2} .
\end{aligned}
$$

In the above equations $b_{i}=s\left(\frac{i}{2 B}\right)$ and $a_{i}=n\left(\frac{i}{2 B}\right)$ where $b_{i} \& a_{i}$ are the samples of signal and noise respectively. The noise sample, $a_{i}$, is the sample of a zero mean white Gaussian random process and $y_{1}$ can be derived from above equation as

$$
\sum_{i=i}^{2 B T}\left(\frac{b_{i}}{\sqrt{N_{0} B}}\right)^{2}=\frac{2 B T \frac{I}{T} \int_{0}^{T} s^{2}(t) d t}{N_{0} B}=2 B T^{*} S N R .
$$

Using a central chi-square distribution with $2 B T$ degrees of freedom, $\frac{2 y_{0}}{N_{0}}$ is expressed as sum of squares of $2 B T$ Gaussian variables with zero mean and unit variance and $\frac{2 y_{1}}{N_{0}}$ has non-central chi-square distribution with the same degree of freedom and $2 B T^{*} S N R$ as non-central parameter [14]. By using the above derived equations, probability of false alarm and probability of miss detection can be calculated as [9]

$$
\begin{aligned}
& P_{f}=P(y>\lambda \mid h=0)=P\left(\frac{2 y_{0}}{N_{0}}>\frac{2 \lambda}{N_{0}}\right), \\
& P_{m}=P(y<\lambda \mid h=1)=P\left(\frac{2 y_{1}}{N_{0}}<\frac{2 \lambda}{N_{0}}\right) .
\end{aligned}
$$

In $p_{f}$, for a given threshold value, the right-hand side shows a chi-square variable with $2 B T$ degree of freedom. Similarly, in the $p_{m}, 2 B T$ shows a non-central chi-square distribution with the non-centrality parameter $2 B T^{*} S N R$. The decision threshold value $\lambda$ is expressed as [9]

$$
\lambda=\beta \cdot B N_{0} \cdot T
$$

where $\beta$ is the normalization factor. Using value of $\lambda$ in the above equations gives [9]

$$
\begin{aligned}
& P_{f}=P\left(\frac{2 \beta \cdot B N_{0} \cdot T}{N_{0}}<\frac{2 y_{0}}{N_{0}}\right), \\
& P_{m}=P\left(\frac{2 \beta \cdot B N_{0} \cdot T}{N_{0}}<\frac{2 y_{I}}{N_{0}}\right), \\
& P_{f}=1-F_{2 B T}(2 B T \beta), \\
& P_{m}=F_{2 B T, 2 B T . S N R}(2 B T \beta) .
\end{aligned}
$$

After calculating $P_{f}$ and $P_{m}$ the energy detection error rate $\left(R_{D E}\right)$ can be defined as

$$
R_{D E}=\alpha P_{m}+(1-\alpha) P_{f}
$$

where $\alpha$ is the wake-up activity rate. According to [9], DER can be reduce by setting threshold so that $\alpha . P_{m}$ and $(1-\alpha) P_{f}$ are equal.

Aloha protocol is used as a channel access method for the wake-up signal transmission process. Simulations have been performed for different number of nodes to find out the channel access success probability when multiple nodes are trying to transmit the WUS signal with a randomly distributed delay between 0 and 1 seconds. The results derived from above PHY analysis and MAC simulations are then used in the network's energy consumption calculation model, as proposed in [7]. This model compares the GWR-MAC and DCM approach. It considers the energy consumption of sensing, processing and communications of both networks. The main energy consumption factors, of each transceiver's component, are: wake-up signaling, data transmission and reception, MCU and sensor active mode current consumption. The total energy consumption during the network operation time, $t$, as a function of number of events and bit error probability for GWR-MAC based network's sensor nodes $(\mathrm{SN})$ and hub $(\mathrm{H})$, can be calculated as [7]

$$
\begin{aligned}
& E_{\mathrm{GWR}-\mathrm{MAC}}^{\mathrm{SN}}(\epsilon, t, \gamma)=E_{\mathrm{s}}^{\mathrm{SN}}(t)+E_{\mathrm{MCU}} \mathrm{SN}(\epsilon, t)+ \\
& E_{\mathrm{TX}, \mathrm{WUS}}(\epsilon, t, \gamma)+E_{\text {wait,BC }}(\epsilon, t)+E_{\mathrm{RX}, \mathrm{BC}}(\epsilon, t) \\
&+ E_{\mathrm{C}}(t)+E_{\mathrm{TX}, \mathrm{DSN}}(\epsilon, t, \gamma)+E_{\mathrm{RX}, \mathrm{ACK}}(\epsilon, t, \gamma)+E_{\mathrm{clk}(t)} \\
& E_{\mathrm{GWR}-\mathrm{MAC}} \mathrm{H}(\epsilon, t, \gamma)=E_{\mathrm{s}}^{\mathrm{H}}(t)+E_{\mathrm{MCU}} \mathrm{H}(\epsilon, t) \\
&+E_{\mathrm{TX}, \mathrm{BC}}(\epsilon, t)+E_{\mathrm{RX}, \mathrm{WUS}}(\epsilon, t, \gamma)+E_{\mathrm{C}}(t) \\
&+ E_{\mathrm{clk}}(t)+E_{\mathrm{RX}, \mathrm{D}} H(\epsilon, t, \gamma)+E_{\mathrm{TX}, \mathrm{ACK}}(\epsilon, t, \gamma),
\end{aligned}
$$


where $\varepsilon$ is the number of events during the operation time $t, \gamma$ is the joint success probability of the PHY \& MAC layers, $E_{\mathrm{TX}}$,wUS and $E_{\mathrm{RX} \text {,WUS }}$ are the energy consumptions of WUS transmissions and reception, respectively. $E_{\mathrm{TX}, \mathrm{BC}}, E_{\mathrm{wait}, \mathrm{BC}}$ and $E_{\mathrm{RX}, \mathrm{BC}}$ are the energy consumptions of $\mathrm{BC}$ transmission, waiting and reception, respectively. $E_{\mathrm{C}}$ is the constant energy consumption of the WUR and $E_{\mathrm{clk}}$ is the energy consumption of the clock needed to maintain the time synchronization. $E^{x}$ is the energy consumption of sensing, $E_{\mathrm{MCU}}^{x}$ is the energy consumption of the MCU, $E^{x} \mathrm{TX, \textrm {D }}$ and $E_{x \mathrm{RX}, \mathrm{D}}$ are the energy consumption of data transmissions and receptions respectively. The model used in this research paper takes into account physical layer's success probability and channel access success probability as these two are vital in any wireless communication and also in calculation of energy consumption. For this research, different parameters settings have been exploited such as decision threshold and wake-up signal-tonoise ratio. The discussed energy consumption model is explained in more details in [7].

\section{RESULTS}

The main parameters used for calculation of performance comparison results are shown in Table 1. These parameters are used to calculate the probabilities of false alarm, miss detection and successful wake-up transmission, which affect the calculation of total energy consumption.

TABLE 1. PARAMETERS FOR TOTAL ENERGY CONSUMPTION

\begin{tabular}{|l|l|l|}
\hline Parameter & Description & Value \\
\hline$N$ & Number of nodes & 10 \\
\hline$\lambda$ & Decision threshold & $1.1,1.2,1.4$ \\
\hline$S N R$ & $\begin{array}{l}\text { Signal-to-noise-ratio for wake-up } \\
\text { signal detection }\end{array}$ & -6 to $4 \mathrm{~dB}$ \\
\hline$B W$ & Bandwidth & $30 \mathrm{MHz}$ \\
\hline$T$ & Integration time & $10 \mu \mathrm{sec}$ \\
\hline
\end{tabular}

MAC simulations are performed to find out successful wake-up probability as a function of packet duration. The results are explored for 2, 5 and 10 nodes cases. For the simulations, the maximum amount of transmission attempt is specified to be 20 . Wake-up probability results are calculated as an average of 2 million simulation rounds. These results are then incorporated in calculation of network's energy consumption. The probabilities of false alarm and miss detection are calculated by using the introduced analytical model and used to find out detection error rate. The result shown in Fig. 2, illustrates that the $R_{D E}$ is high when the wakeup signal-to-noise-ratio is $-6 \mathrm{~dB}$. As the wake-up SNR increases, the DER decreases. Once the SNR reaches $0 \mathrm{~dB}$ the $R_{D E}$ stays constant after that. The result states that the $R_{D E}$ is high for $\lambda=1.4$ in comparison to other threshold values. To keep $R_{D E}$ low, either a high $S N R$ is required or decision threshold value should be kept low.

By utilizing the results of PHY and MAC layer's evaluation, total energy consumption of the network has been calculated. The result shows total energy consumption of DCM

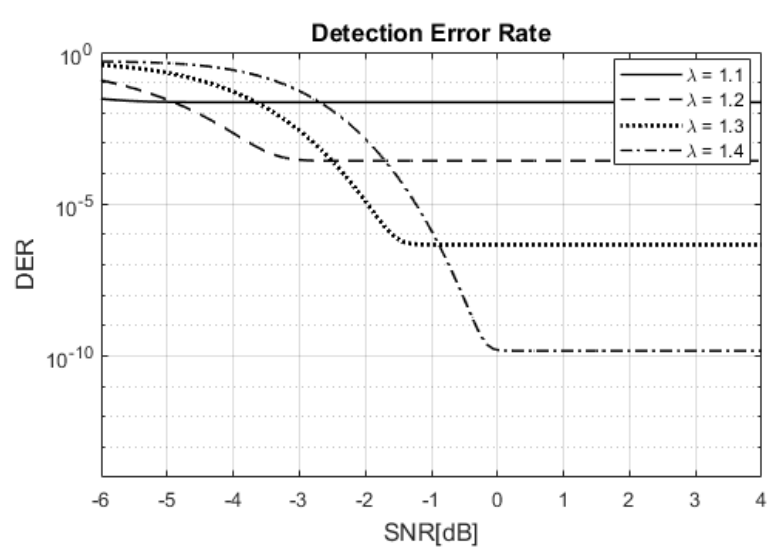

Figure 2. Detection error rate.

and GWR-MAC network as a function of occurrence of events per hour. $E_{b} / N_{0}$ is assumed to be high enough $(>18 \mathrm{~dB})$ for erroneous data transmissions. Therefore, it will not affect to comparison of WUR and DCM networks. Two parametric values of SNR and decision threshold have been exploited to study their effects on the network's total energy consumption. To explore the effects of SNR two results are shown for the cases where decision threshold remains constant (1.2) and the SNR varies. In the first case SNR is $-6 \mathrm{~dB}$ and for the second case it is $-2 \mathrm{~dB}$. It can be seen from Fig. 3 and Fig. 4, that with higher SNR the energy consumption of the WUR based network decreased remarkably. This highlights the importance of high probability of successful wake-up process.

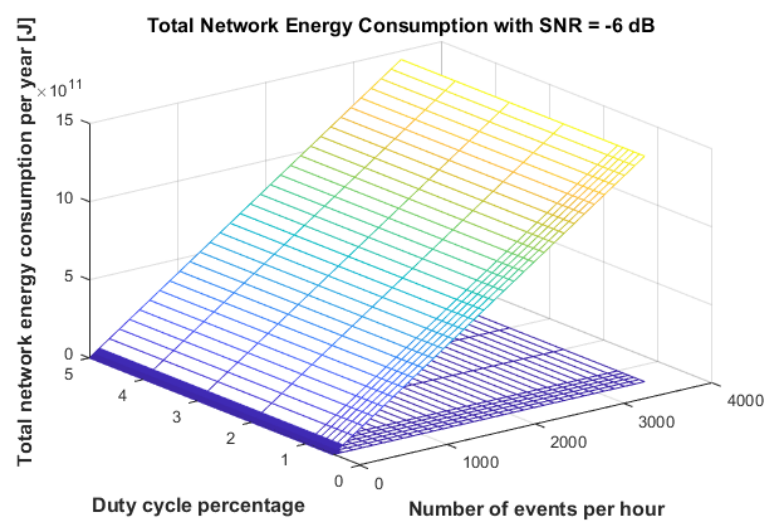

Figure 3. Energy consumption of network with $S N R=-6 \mathrm{~dB}$.

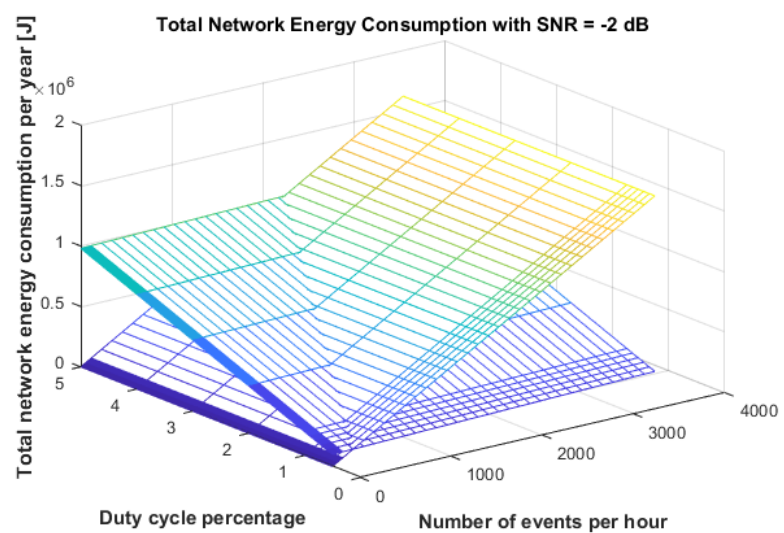

Figure 4. Energy consumption of network with $S N R=-2 \mathrm{~dB}$. 
To illustrate the impact of decision threshold value two cases are shown here by using $\lambda=1.1$ and $\lambda=1.4$. Fig. 5 and Fig. 6 illustrates the total network energy consumption when the wake-up signal $S N R$ is $-2 \mathrm{~dB}$. The result states that as the decision threshold value is increased from 1.1 to 1.4 the total energy consumption of the network also increased in WUR case. It is because for higher decision threshold value the probability of miss detection is high which causes higher energy consumption.

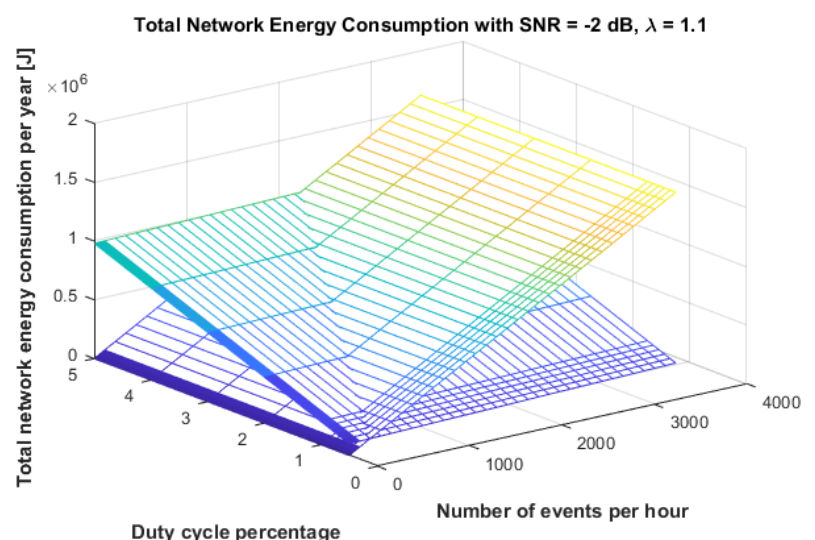

Figure 5. Energy consumption of network with $\lambda=1.1$.

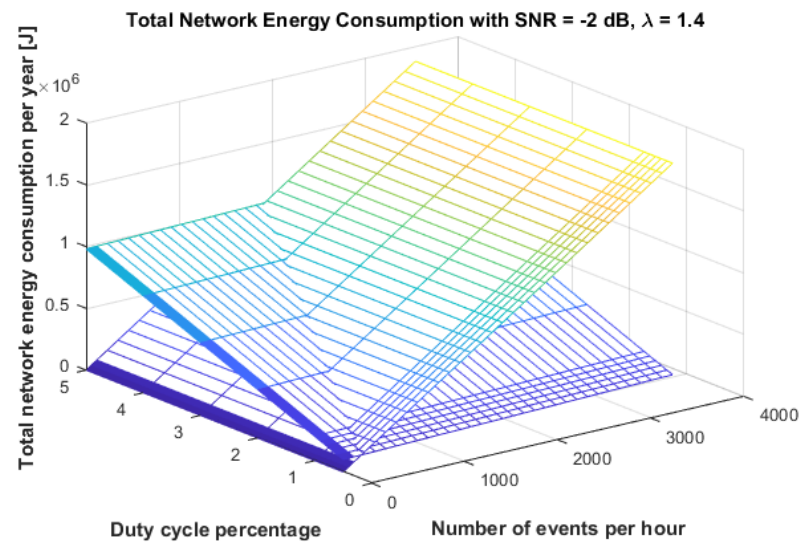

Figure 6. Energy consumption of network with $\lambda=1.4$.

\section{CONCLUSION}

In this work, the focus was on the energy consumption evaluation of GWR-MAC protocol which target to save energy by avoiding idle listening. An analytical model and simulations were used to derive the wake-up process success probability by taking into account PHY and MAC layer aspects. Then the total energy consumption of network was calculated by using different parameters in order to find out how the wake-up process success probability affects to energy efficiency of WUR based networking. It was found that when the wake-up signal-to-noise ratio is sufficient, the WUR based approach is more energy efficient in the target applications which require communication rarely. However, if the wake-up signal-to-noise ratio is low, the total energy consumption of WUR network increases drastically, making it less energy efficient than dutycycling base network. Further, it was found the decision threshold of energy detection based WUS reception has an effect to the energy efficiency superiority between WUR and duty-cycling based approaches. A high successful wake-up transmission probability is vital to keep the total energy consumption of the WUR network low. Total energy consumption of the network varies also with the number of event occurrence, i.e., the frequency of the need for communication. If the network's event occurrence is low, it is better to use WUR base approach to save energy. In the case of very frequent communication need, DCM approach is more energy efficient.

\section{REFERENCES}

[1] Darwish A. and Hassanien A. E., "Wearable and Implantable Wireless Sensor Network Solutions for Healthcare Monitoring," Sensors 2011; 11(6): 5561-5595.

[2] Negra R., Jemili I. and Belghith A., "Wireless Body Area Networks: Applications and Technologies," International Workshop on Recent Advances on Machine-to-Machine Communications, Procedia Computer Science 83, 2016, pp. $1274-1281$.

[3] Javaid N., Hayat S. and Shakir M., "Energy Efficient MAC Protocols in Wireless Body Area Sensor Networks: A Survey," J. Basic Appl. Sci. Res. 20133 (4): 770-778.

[4] Djiroun F. Z. and Djenouri D., "MAC Protocols With Wake-Up Radio for Wireless Sensor Networks: A Review," IEEE Communications Surveys \& Tutorials, vol 19, no 1, pp. 587-618, Firstquarter 2017.

[5] Piyare R., Murphy A. L., Kiraly C., Tosato P. and Brunelli D., "Ultra Low Power Wake-Up Radios: A Hardware and Networking Survey," IEEE Communications Surveys \& Tutorials, July, 2017.

[6] Karvonen H., Petäjäjärvi J., Iinatti J., Hämäläinen M. and Pomalaza-Ráez C., "A Generic Wake-up Radio based MAC Protocol for Energy Efficient Short Range Communication" IEEE International Symposium on Personal, Indoor, and Mobile Radio Communication (PIMRC), 2014, Washington DC, 2014, pp. 2173- 2177.

[7] Karvonen H., Iinatti J., Petäjäjärvi J. and Hämäläinen M., "Energy Efficient IR-UWB WBAN Using a Generic Wake-up Radio Based MAC Protocol," International Conference on Body Area Networks (BodyNets '14), London, Great Britain, September 2014.

[8] Gu L. and Stankovic J. A., "Radio-Triggered Wake-Up Capability for Sensor Networks," IEEE Real-Time and Embedded Technology and Applications Symposium, 2004, pp. 27-36.

[9] Zhou W. and Rabaey J., "Energy Detection Technique for Ultra-Low Power High Sensitivity Wake-Up Receiver," IEEE International Symposium on Circuits and Systems (ISCAS2013), Beijing, China, 2013, pp. 1857-1860.

[10] Magno M. and Benini L., "An Ultra Low Power High Sensitivity WakeUp Radio Receiver with Addressing Capability," IEEE International Conference on Wireless and Mobile Computing, Networking and Communications (WiMob), Larnaca, Cypros, 2014, pp. 92-99.

[11] Marinkovic S. and Popovici E., "Nano-power Wake-Up Radio Circuit for Wireless Body Area Networks," IEEE Radio and Wireless Symposium (RWS), Phoenix AZ, USA, 2011, pp. 398-401.

[12] Miller M. and Vaidya N., "A MAC Protocol to Reduce Sensor Network Energy Consumption Using a Wakeup Radio," IEEE Transactions on Mobile Computing, vol. 4, no. 3 pp. 228-242, May-June 2005.

[13] Jamthe A. and Agrawal D. P., "Approaches for Energy Harvesting and Power Management in Wireless Healthcare Sensor Networks", Int. J. Comput. Commun. Eng., vol. 2, no. 5, pp. 596-600, Sep. 2013.

[14] Urkowitz H., "Energy Detection of Unknown Deterministic Signals," in Proceedings of the IEEE, vol. 55, no. 4, pp. 523-531, April 1967. 